\title{
Single-Electron Shuttle Based on Electron Spin
}

\author{
S. I. Kulinich, ${ }^{1,2,3}$ L. Y. Gorelik, ${ }^{4,}{ }^{*}$ A. N. Kalinenko, ${ }^{1}$ I. V. Krive,,${ }^{1,2,3}$ R. I. Shekhter, ${ }^{2}$ Y. W. Park, ${ }^{3}$ and M. Jonson ${ }^{2,6}$ \\ ${ }^{1}$ B. Verkin Institute for Low Temperature Physics and Engineering of the National Academy of Sciences of Ukraine, \\ 47 Lenin Avenue, Kharkov 61103, Ukraine \\ ${ }^{2}$ Department of Physics, University of Gothenburg, SE-412 96 Göteborg, Sweden \\ ${ }^{3}$ Department of Physics and Astronomy, Seoul National University, Seoul 151-747, Korea \\ ${ }^{4}$ Department of Applied Physics, Chalmers University of Technology, SE-412 96 Göteborg, Sweden \\ ${ }^{5}$ Physical Department, V.N. Karazin National University, Kharkov 61077, Ukraine \\ ${ }^{6}$ SUPA, Institute of Photonics and Quantum Sciences, Heriot-Watt University, Edinburgh EH14 4AS, Scotland, United Kingdom \\ (Received 7 October 2013; revised manuscript received 20 January 2014; published 20 March 2014)
}

\begin{abstract}
A nanoelectromechanical device based on magnetic exchange forces and electron spin flips induced by a weak external magnetic field is suggested. It is shown that this device can operate as a new type of singleelectron "shuttle" in the Coulomb blockade regime of electron transport.
\end{abstract}

DOI: 10.1103/PhysRevLett.112.117206

PACS numbers: $85.85 .+\mathrm{j}, 85.75 .-\mathrm{d}$

Today we are witnessing an interesting development towards spintronics, i.e., electronics based on the interplay between magnetism and the quantum dynamics of the spin of electrons (see the reviews in Ref. [1]). In the context of nanoelectromechanics [2], an interesting question is, therefore, to what extent the spin of the electron, with its typically long coherence times, can replace the charge as a vehicle for electromechanical energy transduction in, e.g., a nanoelectromechanical single-electron transistor (NEM-SET) shuttle device [3,4]. In this Letter we show that the spin can indeed play this role.

Our model "spintro-mechanical" shuttle device, shown in Fig. 1, comprises two ferromagnetic leads (for now assumed to be $100 \%$ spin polarized "half metals" [5]) with antiparallel magnetization and a quantum dot with a single spin-degenerate electronic level. The exchange interaction between the magnetic leads and an electron spin on the dot $[6,7]$ generates a mechanical force that acts on the movable dot. If strong enough, this force can significantly modify electron transport through a mechanically damped shuttle device operating near mechanical equilibrium (with dot vibrations of small amplitude) [8]. Here we will show that for low enough mechanical damping the strongly nonequilibrium phenomenon of single-electron shuttling may be triggered by a weak external magnetic field.

Spin accumulation on the dot can be controlled in two ways: (i) by injecting spin-polarized electrons from the source electrode, and (ii) by flipping the spin on the dot. Below, we will show that a combination of these two mechanisms can lead to a spintro-mechanical instability with respect to the onset of mechanical vibrations of the dot if both a bias voltage (which leads to spin injection) and a weak, perpendicular external magnetic field (which induces spin flips) of appropriate strength are applied.

A particularly transparent picture of how spintromechanics affect shuttle vibrations emerges in the limit of the weak magnetic field $H$ and large electron tunneling rate $\Gamma_{S(D)}$ between dot and source- and drain electrodes. In order to explore this limit, where $\Gamma_{S} \gg \omega \gg(\mu H / \hbar)^{2} / \Gamma_{D}$ and $\omega / 2 \pi$ is the natural vibration frequency of the dot, we focus first on the total work done by the exchange force $\mathfrak{F}$ as the dot vibrates under the influence of an elastic force only. In the absence of an external magnetic field [9] the dot is in this case occupied by a spin-up electron emanating from the source electrode (see Fig. 1). This spin is a constant of motion and hence no electrical current through the device is possible since only spin-down states are available in the drain electrode. During the oscillatory motion of the dot the exchange force is therefore always directed towards the source electrode while its magnitude only depends on the position of the dot, $\mathfrak{F}=\mathfrak{F}_{0}(x)$. As a result, no net work is done by the exchange force on the dot. This is because contributions are positive or negative depending on the direction of the dot's motion and cancel when summed over one oscillation period. A finite amount of work can only be done if the exchange force deviates from $\mathfrak{F}_{0}(x)$ as a result of spin flip processes induced by the external magnetic field. Such a deviation can be viewed as

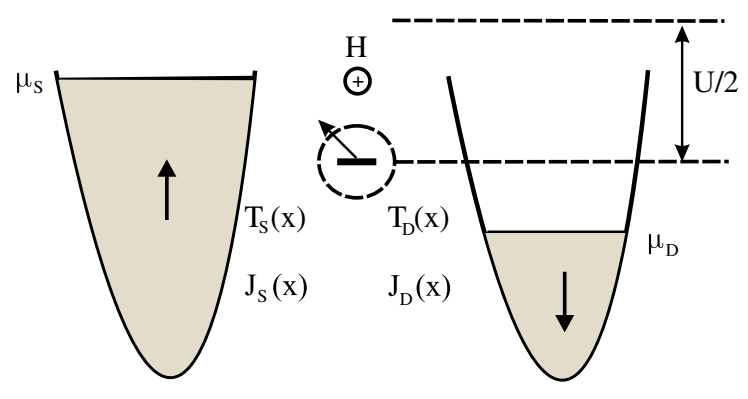

FIG. 1 (color online). Sketch of the nanomagnetic device discussed in the text: a movable quantum dot, modeled as a single spin-degenerate electron level and subject to an external magnetic field, is coupled to two leads with antiparallel magnetization. 
an additional random force $\mathfrak{F}_{H}$ that acts in the opposite direction to $\mathfrak{F}_{0}(x)$. In the limit of a large tunneling rate, $\Gamma_{S(D)} \gg \mu H / \hbar$, and small vibration amplitude a spin flip occurs with a probability $\propto(\mu H / \hbar)^{2} /\left(\omega \Gamma_{D}\right)$ during one oscillation period and is instantly [10] accompanied by the tunneling of the dot electron into the drain electrode, thereby triggering the force $\mathfrak{\Im}_{H}$. The duration of this force is determined by the time $\delta t \sim 1 / \Gamma_{S}(x(t))$ it takes for the spin of the dot to be "restored" by another electron tunneling from the source electrode.

The spin-flip induced random force $\mathfrak{F}_{H}=-\mathfrak{F}_{0}(x)$ is always directed towards the drain electrode. Hence, its effect depends on the dot's direction of motion: as the dot moves away from the source electrode it will be accelerated, while as it moves towards the source it will be decelerated. Since a spin flip may occur at any point on the trajectory one needs to average over different spin-flip positions in order to calculate the net work done on the dot. The result, which depends on the competition between the effect of spin flips that occur at the same position but with the dot moving in opposite directions, is nonzero because $\delta t$ is different in the two cases. As the dot moves away from the source electrode the tunneling rate to this electrode will decrease while as the dot moves towards the source it will increase. This means that the duration of spin-flip induced acceleration will prevail over the one for deceleration. As a result, in weak magnetic fields, the dot will accelerate with time and one can expect a spintro-mechanical shuttle instability in this limit.

The situation is qualitatively different in the opposite limit of strong magnetic fields, where $\Gamma_{S(D)} \ll \mu H / \hbar$ and the spin rotation frequency therefore greatly exceeds the tunneling rates. In this case the quick precession of the electron spin in the dot averages the exchange force to zero if one neglects the small effects of electron tunneling to and from the dot. If one takes corrections due to tunneling into account (having in mind that the source electrode only supplies spin-up electrons) one comes to the conclusion that the average spin on the dot will be directed upwards. This results in a net spintro-mechanical force in the direction opposite to that of the net force occurring in a weak magnetic field limit. As a result, in strong magnetic fields one expects a deceleration of the dot. Therefore, there will be no shuttle instability for such magnetic fields.

As we have discussed above, spin-flip assisted electron tunneling from source to dot to drain in our device results in a magnetic exchange force that attracts the dot to the source electrode. It is interesting to note that this is contrary to the effect of the Coulomb force in the same device [11]. Indeed, since the Coulomb force depends on the electric charge of the dot it repels the dot from the source electrode. Hence, while the dot is empty as the result of a spin-flip assisted tunneling event from dot to drain, an "extra" attractive Coulomb force $\mathfrak{F}_{Q}$ is active. An analysis fully analogous with our previous analysis of the "extra" repulsive magnetic exchange force $\mathfrak{\mho}_{H}$ leads to the conclusion that the effect of the Coulomb force will be just the opposite to that of the exchange force. If the exchange force is sufficiently weak, this means that in the Coulomb blockade regime there is no shuttle instability in the limit of a weak magnetic field, while in strong magnetic fields electron shuttling occurs. Our full analysis below confirms the predictions made above for some limiting cases using only qualitative arguments.

The Hamiltonian $\hat{\mathcal{H}}=\hat{\mathcal{H}}_{l}+\hat{\mathcal{H}}_{d}+\hat{\mathcal{H}}_{v}+\hat{\mathcal{H}}_{t}$ of our system has four terms. The first term, $\hat{\mathcal{H}}_{l}$, describes noninteracting spin polarized electrons in the leads and the last term, $\hat{\mathcal{H}}_{t}$ describes spin-conserving tunneling of electrons between dot and leads with position-dependent tunneling amplitudes $T_{j}(x)=T_{j} \exp (j x / \lambda)$, where $\lambda$ is the dimensionless tunneling length and $j=(S, D)=(-1,+1)$. The second term is the quantum dot Hamiltonian, $\hat{\mathcal{H}}_{d}$, which reads

$$
\begin{aligned}
\hat{\mathcal{H}}_{d}= & \sum_{\sigma=\uparrow, \downarrow} \varepsilon_{0} n_{\sigma}-J_{S}(x)\left(n_{\uparrow}-n_{\downarrow}\right)-J_{D}(x)\left(n_{\downarrow}-n_{\uparrow}\right) \\
& -\frac{g \mu H}{2}\left(a_{\uparrow}^{\dagger} a_{\downarrow}+a_{\downarrow}^{\dagger} a_{\uparrow}\right)-U a_{\uparrow}^{\dagger} a_{\downarrow}^{\dagger} a_{\uparrow} a_{\downarrow} .
\end{aligned}
$$

Here the operator $a_{\sigma}^{\dagger}\left(a_{\sigma}\right)$ creates (destroys) an electron on the dot with energy $\varepsilon_{0}$ and spin $\sigma, n_{\sigma}=a_{\sigma}^{\dagger} a_{\sigma}$ and $J_{j}(x)=$ $J_{j} \exp (j x / \lambda)>0$ is the strength of the ferromagnetic exchange coupling [12], $H$ is the external magnetic field and the intradot electron correlations are characterized by the Coulomb energy $U$. Vibrations of the dot are described by the harmonic oscillator Hamiltonian $\hat{\mathcal{H}}_{v}=(\hbar \omega / 2)\left(p^{2}+x^{2}\right)$, where $[x, p]=i$ and $x$ is the displacement operator for the dot normalized to its zeropoint oscillation amplitude $x_{0}=\sqrt{\hbar / m \omega}, m$ being the mass of the dot. The electrons in each lead are held at a constant electrochemical potential $\mu_{S, D}=\mp e V / 2$ (relative to the Fermi level), where $V \gg\left|2 \varepsilon_{0} / e\right|$ is the bias voltage. We restrict ourselves to the Coulomb blockade regime, $U \sim e^{2} / 2 C>\left|e V / 2-\varepsilon_{0}\right|$ (where $C$ is the capacitance of the dot) and zero temperature [13], so that double occupancy of the dot is forbidden. The electron density of states $\nu_{j}=\nu$ in the leads is assumed to be independent of energy. In what follows we will consider the symmetrical case, $T_{S}=T_{D} \equiv T, J_{D}=J_{S} \equiv J$.

The coherent dynamics of our system is governed by the Hamiltonian $\hat{\mathcal{H}}$ and involve the quantum evolution of the states of the vibrational, charge and spin degrees of freedom. The corresponding set of equations for the density matrix of the mechanical subsystem can be derived, following Refs. [14] and [15], by treating the tunneling Hamiltonian as a small perturbation [16]. Using this approach one can show that without a magnetic field the mechanical subsystem is characterized by an equilibrium density matrix that describes small-amplitude oscillations of the dot in the vicinity of the minima of the potential 
energy. However, when a weak magnetic field $H$ is applied this state becomes unstable with respect to the onset of oscillations with an increasing amplitude if double occupancy of the dot is forbidden by a Coulomb blockade. This instability eventually leads to a stationary state of the shuttling dynamics [17], which can be conveniently analyzed by using the Wigner function representation of the density operators [14]. This approach allows one to calculate the Wigner distribution function $W_{\rho}(x, p)$ for the vibrational degree of freedom to lowest order in the small parameters $1 / \lambda$ and $\alpha=2 J / \lambda \hbar \omega>0$, where $\alpha$ measures the ratio of the exchange force and the mechanical restoring force corresponding to a displacement $x_{0}$. We find [18] that the relevant Wigner function,

$$
W_{\Sigma}^{(0)}(A)=\frac{1}{2 \pi} \int_{0}^{2 \pi} d \varphi W_{\rho}(A \sin \varphi, A \cos \varphi),
$$

which gives the probability distribution of the vibrational amplitudes $A$ in a stationary regime, for $A \ll \lambda$ solves the stationary Fokker-Planck equation

$$
\frac{\partial}{\partial A}\left(A D_{0} \frac{\partial W_{\Sigma}^{(0)}}{\partial A}\right)-\frac{\partial}{\partial A}\left(A^{2} D_{1} W_{\Sigma}^{(0)}\right)=0 .
$$

Here the drift and diffusion coefficients contain the factors

$$
\begin{gathered}
D_{1}=\frac{\alpha}{\lambda} \frac{h^{2} \Gamma^{3}}{\Gamma^{2}+3 h^{2}} \frac{3 \Gamma^{2}+3-h^{2}}{Q_{0}(\Gamma, h)} \text { and } \\
D_{0}=\frac{h^{2} \Gamma}{\Gamma^{2}+3 h^{2}}\left[\frac{\alpha^{2} Q_{1}(\Gamma, h)+\lambda^{-2} Q_{0}(\Gamma, h)}{2 Q_{0}(\Gamma, h)}\right],
\end{gathered}
$$

respectively, where

$$
\begin{gathered}
Q_{0}(\Gamma, h)=\left(1-h^{2}-2 \Gamma^{2}\right)^{2}+\frac{\Gamma^{2}}{4}\left(\Gamma^{2}+3 h^{2}-5\right)^{2}, \\
Q_{1}(\Gamma, h)=\left(1+\frac{9 \Gamma^{2}}{4}\right)\left(1+h^{2}+2 \Gamma^{2}\right)-\frac{5 \Gamma^{4}}{4} .
\end{gathered}
$$

In Eqs. (4-7) all energies have been normalized with respect to the energy quantum $\hbar \omega$ of the mechanical vibrations: $\hbar \omega \rightarrow 1, g \mu H / \hbar \omega \rightarrow h, \Gamma / \omega \rightarrow \Gamma\left(\hbar \Gamma=2 \pi \nu|T|^{2}\right.$ is the level width).

For $A \ll 1$ the solution of Eq. (3) takes the form of a Boltzmann distribution function, $W_{\Sigma}^{(0)} \sim \exp (-\beta \mathcal{E})$, where $\mathcal{E}=A^{2} / 2$ is the dot's vibrational energy and $1 / \beta$, with

$$
\beta=\left(\frac{2 \alpha \Gamma^{2}}{\lambda}\right) \frac{h^{2}-3 \Gamma^{2}-3}{\alpha^{2} Q_{1}(\Gamma, h)+\lambda^{-2} Q_{0}(\Gamma, h)},
$$

is an effective temperature. Since the functions $Q_{0}$ and $Q_{1}$ are positive, the sign of the effective temperature is determined by the relation between the magnetic field, level width, and vibration quantum. In particular, the effective temperature is negative at small magnetic fields, $|H|<H_{c}$, where (reverting to dimensional variables) $g \mu H_{c}=\hbar \sqrt{3\left(\Gamma^{2}+\omega^{2}\right)}$.

A negative $\beta$ implies that the static state of the dot $(A=0)$ is unstable and that a shuttling regime of charge transport $(A \neq 0)$ is realized. It is interesting to note that $\beta$ is finite even as $h \rightarrow 0$. This apparent paradox may be resolved by considering the Fokker-Plank equation in its time-dependent form and noting that the rate of change of the oscillation amplitude at the instability is defined by the coefficient $D_{1}$. This coefficient scales as $D_{1}(h) \propto h^{2}$ as $h \rightarrow 0$ and therefore the shuttle phase is only realized formally after an infinitely long time in this limit. As a function of magnetic field $D_{1}$ has a maximum, $D_{1}^{\max }=0.6(\alpha / \lambda) \Gamma^{-1}$, at $h_{\mathrm{opt}}=0.4 \Gamma$. Therefore, optimal magnetic fields are in the range $0.1-1 \mathrm{~T}$ if $\hbar \Gamma=10-100 \mu \mathrm{eV}$. For high magnetic fields, $|H|>H_{c}$, there is no shuttling regime (at least not with a small vibration amplitude, $A \ll 1$ ) and the vibronic regime, corresponding to small fluctuations of the quantum dot around its equilibrium position, is stable.

The amplitude of the shuttle vibrations that develop as the result of an instability is still described by Eq. (3) for the Wigner distribution function. However, for large amplitudes, $A \gtrsim 1$, the drift- and diffusion coefficients $A^{2} D_{1}$ and $A D_{0}$ can no longer be evaluated analytically. Fortunately, it is sufficient to know the amplitude and magnetic field dependence of $D_{1}$ for a qualitative analysis. This is because a positive value of the drift coefficient means that energy is pumped into the dot vibrations, while a negative value corresponds to damping of the vibrations. Therefore, magnetic fields for which $D_{1}(A)=0$ and $D_{1}^{\prime}(A)<0$ correspond to a stable stationary state of the dot and a local maximum of the Wigner function. Based on this picture one concludes [18] that at low magnetic fields, $h<h_{c 1}$, a shuttling regime with a large vibration amplitude is realized, while at high magnetic fields, $h>h_{c 1}$, the situation is more complicated. Here one of two $\left(h_{c 1}<h<h_{c 2}\right)$ or three $\left(h>h_{c 2}\right)$ shuttling regimes with different amplitudes can be stable depending on the initial conditions (see also Ref. [15]). If the dot is initially in the static state $(A=0)$ a stable shuttle regime only appears for $h<h_{c}$, as already mentioned.

The electron-spin induced spintro-mechanical coupling discussed above is complementary to the conventional electron-charge induced electromechanical coupling. In order to compare their relative importance we have extended the studies in Ref. [15] to the Coulomb blockade regime, $e V<U$, that is of interest here. We find that in the limit $\Gamma \gg 1$ and for small oscillation amplitudes $A$ the drift coefficient induced by the electromechanical coupling has the form $A^{2} D_{1}^{(e)}$ with

$$
D_{1}^{(e)}=-\frac{d}{\alpha \Gamma^{2}} \frac{\left(2 h^{2}-\Gamma^{2}\right)\left(h^{2}+\Gamma^{2}\right)}{h^{2}-3 \Gamma^{2}} D_{1},
$$




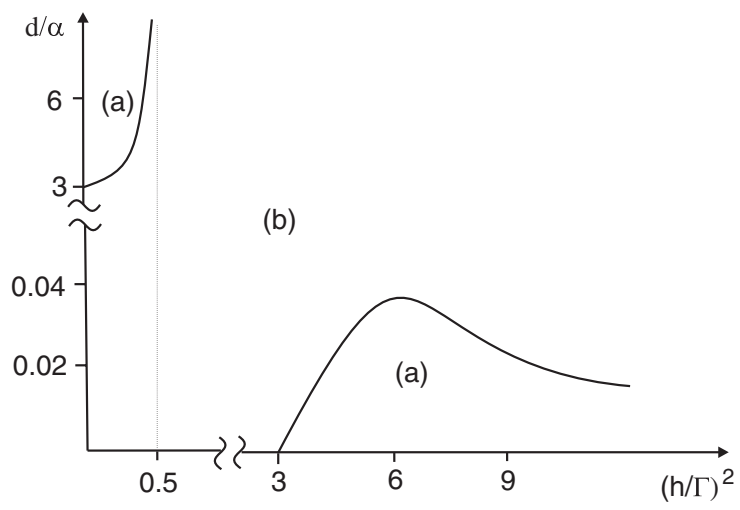

FIG. 2. Shuttle stability diagram in a plane spanned by $d / \alpha=$ $e \mathcal{E} \lambda / 2 J$ and $(h / \Gamma)^{2}$; regions where the static state $(A=0)$ is stable are marked by (a) while the region where shuttling occurs $(A \neq 0)$ is marked by $(b)$.

where $D_{1}$ is given by Eq. (4), $d=e \mathcal{E} x_{0} / \hbar \omega$, and $\mathcal{E}$ is the electric field induced by the potential drop between the leads. Therefore, if $h \ll \Gamma$, the electromechanical coupling -in agreement with our initial qualitative analysisgenerates a negative contribution to the drift coefficient and hence additional damping in the mechanical subsystem. In the limit of small magnetic fields a shuttle instability still occurs if $d<3 \alpha$, i.e., if $e \mathcal{E}<6 J / \lambda$. However, since $\mathcal{E} \propto V$ the electromechanical coupling must dominate above some critical voltage $V_{c}$ [19] and suppress the shuttle regime. In the limit of high magnetic field, where the spintro-mechanical coupling increases the damping in the mechanical subsystem, Fig. 2 shows that the electromechanical coupling generates a shuttle instability for high enough electric fields.

Obviously, a shuttle instability can only occur if more energy is pumped into the mechanical subsystem than it dissipates to the environment. Dissipation can readily be taken into account phenomenologically by including a Lindblad term [14] in the equation of motion for the density matrix. The result is a renormalization of the drift coefficient $D_{1}$ of Eq. (4) that changes the instability criterion from $A^{2} D_{1}>0$ to $A^{2} D_{1}>\gamma$, where $\gamma$ is the Lindblad dissipation coefficient. For the maximum value $D_{1}^{\max }$, which as shown above obtains for $h_{\mathrm{opt}}=0.4 \Gamma$, the latter criterion for a spintromechanical shuttle instability leads to the condition $Q>\left(\lambda / x_{0}\right)^{2}(\hbar \Gamma / J)=m \omega \Gamma \lambda^{2} / J$ for the quality factor $Q \sim 1 / \gamma$. If the condition that the leads are $100 \%$ spin polarized $(\eta=1)$ is relaxed, additional dissipation will be induced due to spin-conserving transitions from source to drain. As a result, the drift coefficient will scale as $\eta A^{2} D_{1}$ with $\eta<1$, which will require a correspondingly larger quality factor for the instability to occur.

An experimental setup such as the one used in Ref. [20] to study Kondo-assisted tunneling via a $\mathrm{C}_{60}$ molecule would seem well suited to observe the phenomenon discussed above. In that experiment one found that coupling to two ferromagnetic nickel electrodes produced a local magnetic exchange field on the $\mathrm{C}_{60}$-quantum dot in excess of $50 \mathrm{~T}[J \sim 2.5 \mathrm{meV}]$ with $\hbar \Gamma \sim 30 \mathrm{meV}$. In a similar setup, but with nonmagnetic electrodes, nanomechanical oscillations of a $\mathrm{C}_{60}$ molecule trapped in an approximately $1 \mathrm{~nm}$ wide gap were detected with a frequency of about $1.2 \mathrm{THz} \quad(\hbar \omega \sim 5 \mathrm{meV})$ [21]. Therefore, taking $\lambda \sim 0.1 \mathrm{~nm}$ and $\eta \sim 0.1$, we predict that by applying a weak magnetic field one can generate mechanical oscillations with a frequency of the order of $1 \mathrm{THz}$ and an amplitude of order $\lambda \sim 0.1 \mathrm{~nm}$ if the quality factor $Q$ is larger than 100 .

In summary, we have shown that a nanoelectromechanical device based on magnetic exchange forces and electron spin flips, induced by a weak external magnetic field, can operate as a single-electron shuttle. By using a simple model for charge transport through a single-level vibrating quantum dot placed between two magnetic leads, we have demonstrated that a shuttle regime of electron transport is realized at low magnetic fields $(\mu H \ll \hbar \Gamma$ in the adiabatic limit $\Gamma \gg \omega)$. Under these conditions the corresponding electrically driven shuttle is still in a vibronic phase (small fluctuations around its equilibrium position). The high sensitivity of the suggested nanoelectromechanical device to a weak external magnetic field, makes the "magnetic shuttle" a promising tool for studying quantum operations in the context of spintronics.

Financial support from the Swedish VR, the National Academy of Science of Ukraine (4/12-N) and the Leading Foreign Research Institutes Recruitment Program (200900514) of NRF, Korea, is gratefully acknowledged. I. K. and S. K. thank the Department of Physics at the University of Gothenburg and the Department of Physics and Astronomy at Seoul National University for their hospitality.

*gorelik@chalmers.se

[1] I. Zutic, J. Fabian, and S. Das Sarma, Rev. Mod. Phys. 76, 323 (2004); D. D. Awschalom, L. C. Bassett, A. S. Dzurak, E. L. Hu, and J. R. Petta, Science 339, 1174 (2013).

[2] M. Poot and H. S. J. van der Zant, Phys. Rep. 511, 273 (2012).

[3] L. Y. Gorelik, A. Isacsson, M. V. Voinova, B. Kasemo, R. I. Shekhter, and M. Jonson, Phys. Rev. Lett. 80, 4526 (1998).

[4] R. I. Shekhter, Yu. Galperin, L. Y. Gorelik, A. Isacsson and M. Jonson, J. Phys. Condens. Matter 15, R441 (2003); R. I. Shekhter, L. Y. Gorelik, I. V. Krive, M. N. Kiselev, A. V. Parafilo, and M. Jonson, Nanoelectromechanical Systems 1, 1 (2013).

[5] M. Ziese, Rep. Prog. Phys. 65, 143 (2002).

[6] Y. K. Takahashi, S. Kasai, T. Furubayashi, S. Mitani, K. Inomata, and K. Hono, Appl. Phys. Lett. 96, 072512 (2010).

[7] E. Wada, K. Watanabe, Y. Shirahata, M. Itoh, M. Yamaguchi, and T. Taniyama, Appl. Phys. Lett. 96, 102510 (2010). 
[8] R. I. Shekhter, A. Pulkin, and M. Jonson, Phys. Rev. B 86, 100404(R) (2012).

[9] For the moment, we also neglect mechanical damping and intrinsic spin-flip mechanisms.

[10] The first nonvanishing term in a perturbation expansion of the tunneling probability is of $\operatorname{order}\left(\mu H / \Gamma_{D}\right)^{2}$ and corresponds to spin-flip assisted tunneling.

[11] We note the important role of the Coulomb blockade, which prevents double occupancy of the dot and makes it necessary for one electron to tunnel to the drain before another can enter from the source. This means that in between these events the dot has a deficit of electron charge. Without a Coulomb blockade (as in Ref. [15]) there is no restriction on the tunneling sequence and the dot may have a deficit or a surplus of electrons depending on the sequence of tunneling to and from the dot. As it turns out, this difference leads to opposite conclusions concerning the current-induced mechanical stability of the device in Ref. [15] and this work in the limit of finite electric force and no exchange interaction.

[12] The magnitude $J_{j}(x)$ and $T_{j}(x)$ both depend on the overlap of wave functions in a lead and the dot. We use the same widely accepted phenomenological model for both.

[13] A finite temperature $T$ will obviously not affect the electron dynamics if $k_{B} T \ll\left|e V / 2-\varepsilon_{0}\right|$.

[14] T. Novotny, A. Donarini, and A.-P. Jauho, Phys. Rev. Lett. 90, 256801 (2003); D. Fedorets, L. Y. Gorelik, R. I. Shekhter, and M. Jonson, Phys. Rev. Lett. 92, 166801 (2004).
[15] D. Fedorets, L. Y. Gorelik, R. I. Shekhter, and M. Jonson, Phys. Rev. Lett. 95, 057203 (2005); L. Y. Gorelik, D. Fedorets, R. I. Shekhter, and M. Jonson, New J. Phys. 7, 242 (2005).

[16] A. Mitra, I. Aleiner, and A. J. Millis, Phys. Rev. B 69, 245302 (2004).

[17] Our numerical analysis shows that the exponential increase of the vibration amplitude in the linear regime stabilizes at higher amplitudes due to nonlinear effects even without dissipation [18]. This is in contrast to the shuttle instability found in the absence of a Coulomb blockade, where a finiteamplitude stationary state is only achieved if there is dissipation [15].

[18] See Supplemental Material at http://link.aps.org/ supplemental/10.1103/PhysRevLett.112.117206 where we derive and solve the Fokker-Planck equation for the Wigner distribution functions used in this Letter for analyzing a nanomagnetic shuttle device.

[19] If $J=10-100 \mu \mathrm{eV}$ and $\lambda \sim 0.1 \mathrm{~nm}$ one finds that $V_{c} \sim$ 6-60 meV if the source and drain electrodes are $10 \mathrm{~nm}$ apart.

[20] A. N. Pasupathy, R. C. Bialczak, J. Martinek, J. E. Grose, L. A. K. Donev, P. L. McEuen, and D. C. Ralph, Science 306, 86 (2004).

[21] H. Park, J. Park, A. K. L. Lim, E. H. Anderson, A. Paul Alivisatos, and P. L. McEuen, Nature (London) 407, 57 (2000). 